\title{
Design and Deployment of a Pediatric Cardiac Arrest Surveillance System
}

\author{
Jordan Michel Duval-Arnould $\left(D^{1},{ }^{1}\right.$ Heather Marie Newton, ${ }^{2}$ Leann McNamara, ${ }^{3}$ \\ Branden Michael Engorn, ${ }^{4}$ Kareen Jones, ${ }^{5}$ Meghan Bernier, ${ }^{5}$ Pamela Dodge, ${ }^{6}$ \\ Cheryl Salamone, ${ }^{7}$ Utpal Bhalala, ${ }^{5}$ Justin M. Jeffers, ${ }^{6}$ Lilly Engineer, ${ }^{5}$ \\ Marie Diener-West, ${ }^{8}$ and Elizabeth Anne Hunt ${ }^{1}$
}

\author{
${ }^{1}$ Division of Health Sciences Informatics, Department of Anesthesiology and Critical Care Medicine, School of Medicine, \\ The Johns Hopkins University, Baltimore, MD, USA \\ ${ }^{2}$ Department of Occupational Health, The Johns Hopkins Hospital, Baltimore, MD, USA \\ ${ }^{3}$ Department of Pharmacy, The Johns Hopkins Hospital, Baltimore, MD, USA \\ ${ }^{4}$ Department of Anesthesiology and Critical Care Medicine and Department of Pediatrics, School of Medicine, \\ The Johns Hopkins University, Baltimore, MD, USA \\ ${ }^{5}$ Department of Anesthesiology and Critical Care Medicine, School of Medicine, The Johns Hopkins University, \\ Baltimore, MD, USA \\ ${ }^{6}$ Department of Pediatrics, The Johns Hopkins Hospital, Baltimore, MD, USA \\ ${ }^{7}$ Neonatology Respiratory Therapy, The Johns Hopkins Hospital, Baltimore, MD, USA \\ ${ }^{8}$ Department of Biostatistics, Johns Hopkins University Bloomberg School of Public Health, Baltimore, MD, USA
}

Correspondence should be addressed to Jordan Michel Duval-Arnould; jordan@jhu.edu

Received 14 November 2017; Accepted 27 March 2018; Published 9 May 2018

Academic Editor: Thomas J. Esposito

Copyright (C) 2018 Jordan Michel Duval-Arnould et al. This is an open access article distributed under the Creative Commons Attribution License, which permits unrestricted use, distribution, and reproduction in any medium, provided the original work is properly cited.

\begin{abstract}
Objective. We aimed to increase detection of pediatric cardiopulmonary resuscitation (CPR) events and collection of physiologic and performance data for use in quality improvement (QI) efforts. Materials and Methods. We developed a workflow-driven surveillance system that leveraged organizational information technology systems to trigger CPR detection and analysis processes. We characterized detection by notification source, type, location, and year, and compared it to previous methods of detection. Results. From 1/1/2013 through 12/31/2015, there were 2,986 unique notifications associated with 2,145 events, 317 requiring CPR. PICU and PEDS-ED accounted for $65 \%$ of CPR events, whereas floor care areas were responsible for only $3 \%$ of events. $100 \%$ of PEDS-OR and $>70 \%$ of PICU CPR events would not have been included in QI efforts. Performance data from both defibrillator and bedside monitor increased annually. (2013: 1\%; 2014: 18\%; 2015: 27\%). Discussion. After deployment of this system, detection has increased $~ 9$-fold and performance data collection increased annually. Had the system not been deployed, 100\% of PEDS-OR and $50-70 \%$ of PICU, NICU, and PEDS-ED events would have been missed. Conclusion. By leveraging hospital information technology and medical device data, identification of pediatric cardiac arrest with an associated increased capture in the proportion of objective performance data is possible.
\end{abstract}

\section{Background and Significance}

Cardiac arrest (CA) is a fatal medical condition as well as a significant public health challenge. The most recent estimates suggest that in the United States (U.S.), approximately 5,00,000 adults and children experience a cardiac arrest annually; globally the number is in the millions. In the U.S., survival from cardiac arrest is less than $15 \%[1-5]$. Though less common in children than adults, pediatric sudden cardiac arrest incidence, case fatality, and years of potential life lost are all significant.

Literature published at the turn of the century suggests that pediatric cardiac arrests occur in $0.7 \%$ to $3 \%$ of pediatric 
hospital admissions and $1.8 \%$ to $5.5 \%$ of pediatric intensive care unit (PICU) admissions [6-9]. Nation-wide 4,000 pediatric in-hospital cardiac arrests (IHCAs) per year require at least two minutes of CPR [10]. A recent multicenter study suggests that less than $25 \%$ pediatric IHCAs require less than 2 minutes [11]; evaluation of 5 years of epidemiologic data from this institution suggests this could be as high as $37 \%$ (165/446). The PICU estimates suggest at least one cardiac arrest per 100 admissions [12]. Recent estimates suggest that the number of annual PICU admissions is between 2,30,000 and 4,10,000, resulting in a possible annual range of cardiac arrests from 2,300 to 4,100 in PICUs in the U.S. [13, 14].

Pediatric in-patients are increasingly monitored by a variety of methods, along with regular and frequent interaction by a range of providers throughout the course of their care. Despite this high degree of electronic and human monitoring, when an IHCA occurs, awareness of the event may be limited to the primary team involved with the patient. Individuals who are distant from the event, whether it be geographically (the other side of the unit/building), temporally (the next day), or institutionally (another department), may be unaware that the event even occurred.

At this institution, measurement of the incidence of "true cardiac arrests" was a perpetual and particularly stubborn challenge, and accurate statistics were essentially nonexistent. Despite electronic health record (EHR) documentation, participation in a large cardiac arrest and CPR registry, dedicated resources to abstract and enter data, internal emergency response teams, bedside monitors and "code blue" buttons, surveillance of these events was limited due to the manual approach for event detection rather than the use of automated or semiautomated methods.

Identification of cardiac arrest events outside of the Pediatric Intensive Care Unit (PICU) usually took place when the Pediatric Rapid Response Team (RRT) was called. At this point, the event was documented on a standardized paper flow sheet and eventually handed to the organizational group responsible for event accounting. Pediatric cardiac arrest is rare in general care settings [15] and tends to occur less frequently on the wards versus other critical care locations such as the Pediatric Emergency Department (PEDSED), PICU, operating room (OR), and magnetic resonance imaging (MRI) procedures areas [16]. In these areas, the RRT is seldom activated, because the teams trained in acute pediatric resuscitation were already physically present. In the MRI procedure areas, it is standard practice for either a critical care nurse, and/or fellow, and/or respiratory staff to accompany the patients needing these procedures. It is also very common for an attending anesthesiologist to be present performing related tasks involved in the procedure, and thus the identification mechanism is often not triggered, given the availability of advanced practice providers and staff. Also, in most PICUs, PEDS-EDs, and ORs, there are unitspecific code alerts and code teams. This reduces the likelihood that these acute and critical care area events will be detected, documented, and discussed as part of evidencebased quality improvement initiatives at an institutional level. In order for all events to be captured, an active and reliable surveillance system is needed.
In April 2012, this institution's entire pediatric population was moved into a new clinical building. Despite the advanced technology present in the building, including integrated nurse call, code blue button, and electronic paging systems, there was no increase in the detection of CA events. These systems, among others, were not being leveraged to serve as notification sources of possible cardiac arrest for use as part of an active surveillance system. As a result, valuable data captured by advanced bedside monitors and smart defibrillators (those that display and record patient and performance data during CPR) were not being collected or evaluated and ultimately not being used to benefit providers or future patients.

This loss of data and underidentification of pediatric CA events represent significant missed opportunities for learning, performance improvement, and contribution to a larger body of scientific knowledge. These opportunities align directly with the National Academies' 2015 recommendations as described in "strategies to improve cardiac arrest survival" including the need for comprehensive surveillance, the need for robust data collection and dissemination, and improvement of the delivery of high-quality resuscitation [17].

\section{Objective}

The aim of this study is to increase the detection of pediatric CPR resuscitation events and collection of physiologic and performance data through the implementation of partially automated, workflow-driven CA surveillance system.

\section{Materials and Methods}

3.1. Process Flow: Resuscitation Event Analysis Clearinghouse (REACH) Surveillance System. After IRB approval, existing information systems utilized on the medical campus, and specifically, at the onset of CA were identified as surveillance notification data sources. These systems were configured to automatically message the implemented computer-based surveillance system known by the acronym "REACH" each time they were activated, providing information regarding date, time, and location of event. The system attempted to capture any possible pediatric CA (defined as a child who received chest compressions and/or defibrillation). Logic was developed to screen notifications as they were added to the system by a combination of factors including geographic origin and message text; these were iteratively refined in order to automatically differentiate potential pediatric events versus those events as definitely adult in nature. This institution is not a free-standing pediatric facility but rather a children's center further integrated in a larger medical campus. Pediatric notifications were automatically disseminated to a 60 person multidisciplinary quality improvement (MDQI) group via email as a "potential CA." Membership of the MDQI included representatives from all hospital care areas, and included residents, fellows, faculty, nurses, respiratory therapists, and pharmacists. The flow to this point was fully automated, standardized, and required no human intervention since it was driven by preexisting provider-based task workflow (Figure 1). 


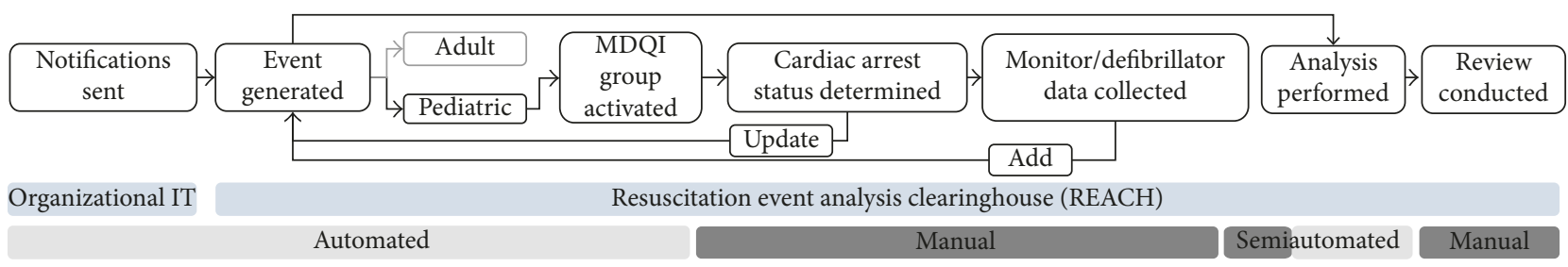

Figure 1: Conceptual model of pediatric cardiac arrest surveillance system process flow.

The manual part of the process began when the MDQI group received notifications and determination of CA status took place. When a true CA was verified, these events were designated as such in REACH and data collection of bedside monitor and smart defibrillator data was initiated. Notification, event, physiologic, and performance data were analyzed and made available for review through different organizational mechanisms. The technology acceptance model informed the overall design process [18]. We considered maximizing the perceived ease of use through the integration of preexisting workflows and organizational IT systems. We aimed to maximize the system's perceived usefulness by providing data in live-time to the group regarding the event status (i.e., improved situational awareness regarding acute events in the hospital) as well as postevent performance assessment based on data obtained upon successful completion of the detection process.

\subsection{Design: Surveillance System Components}

(A) Organizational IT Systems. It is used at the onset or during cardiac arrest and capable of sending email messages. The ability of every system identified to send email messages in the periarrest period largely drove the design decision to leverage email as the messaging protocol for notifications. Appendix A.1 describes each system and configuration considerations necessary for integration.

(B) Organizational Enterprise Email. It receives messages from the organizational IT systems. Based on the sender, it flags messages as valid for consumption by polling service. It uses rule-based processing to identify likely pediatric-related notifications. It relays pediatric notifications to MDQI listserv.

(C) Relational Database. It stores the following: user information, notification, event, CPR, and various rules, preference, and usage data.

(C1). It is automatically backed up fully every 24 hours and differentially every 1 hour.

(D) System Logic and User Interface. It provides management of notification, event, and monitor/smart defibrillator records and data analysis features.

(E) Listserv. It includes the members of the MDQI group. This list is the primary method by which cardiac arrest status communicated amongst the group once determined.
(F) Polling Service. It queries the organizational email, retrieves and extracts new notification message data, and standardizes and inserts in database (Figure 2).

3.3. Deployment. The system was developed over a six-month period, tested for two months, and launched on January 1, 2013; reporting and analysis features were added in 2014. As notifications were generated and sent to the system, each was associated with an event; multiple notifications could be linked to the same event. An event location was associated with one of the 10 possible care areas (Figure 3) and designated as a CA event or not. For events that were designated CA, if smart defibrillator and/or bedside monitor data were collected these were added to the event record.

\section{Results}

4.1. Surveillance System Identification and Detection of Events. For the period 1/1/2013 through 12/31/2015, there were 2,986 unique notifications (i.e., the triggering of a notification source: Figure 2 "A" or Appendix A.1) associated with 2,145 events, of which 317 were designated as CA requiring chest compressions and/or defibrillation (Figure 4). There were 1.4 notifications per event (range: 1-7). Seventy percent (70\%) of events had three or less notifications. Only three events had the maximum (seven) notifications observed; all were CA events. CA events made up approximately $15 \%$ of all events detected by the surveillance system.

4.2. Notifications, Events, and CA by Care Area. The PICU, floor, and PEDS-ED were the top three notification generators with 983,1030 , and 365 , respectively (Table 1). These care areas also had the most events (PICU : 854, floor:512, PEDS-ED:315). The PICU and PEDS-ED accounted for $65 \%$ of all CA events, whereas floor care areas experienced $3 \%$ of all pediatric CA events.

Approximately $20 \%$ of PICU and PEDS-ED notifications and events were CA-related (Table 2). The proportion of PEDS-OR events and notifications that were CA-related was $32 \%$, and the NICU and Imaging-diagnostic care areas had 30 and $37 \%$ of events being CA-related, respectively; $40 \%$ of notifications were CA-related for both areas.

4.3. Differences in Notifications per Event Given CA Event Status. When comparing the total number of notifications per event by CA status, there was a statistically significant difference between the two groups (CA: 1.6 versus non-CA: 1.4; $p<0.001$; Wilcoxon rank-sum) (Table 3). 


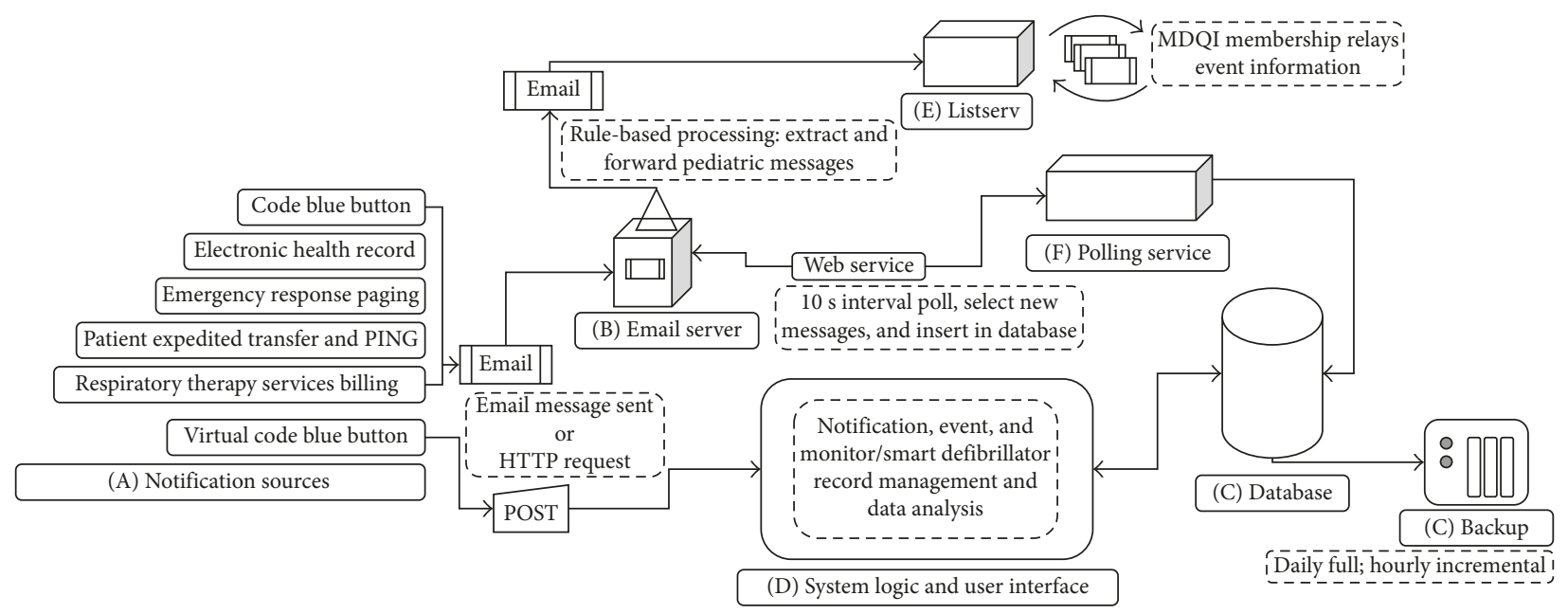

Figure 2: Pediatric cardiac arrest surveillance system data flow.

\begin{tabular}{lcc}
\hline Care area & Description & Children's center \\
\hline Clinic & Outpatient treatment areas & Yes \\
Floor & Inpatient nonacute and noncritical care areas & Yes \\
Imaging-diagnostic & CT, MRI, and X-ray & Yes \\
NICU & Neonatal ICU & Yes \\
Non-children's hospital & On the medical campus and clinical or nonclinical area & No \\
Other children's hospitals & Nonclinical area & Yes \\
PACU & Perianesthesia care area & Yes \\
PEDS-ED & Pediatric emergency department & Yes \\
PEDS-OR & Pediatric operating rooms & Yes \\
PICU & Pediatric ICU (cardiac and noncardiac) & Yes \\
\hline
\end{tabular}

FIgURE 3: Notification and event location types.

Notifications

Figure 4: Aggregate and annual frequency of pediatric notifications, events, and cardiac arrests.

4.4. Surveillance Performance. Examining the type of notification sources associated with each CA event allowed for the determination of whether the event would have been detected had the surveillance system not been put in place (Table 4). Events were flagged as having been identified by any other source other than the EHR or the emergency response paging (methods available and utilized prior to the deployment of REACH). One-hundred percent of the PEDSOR CA events would not have been detected and/or reported. Over $70 \%$ of PICU and approximately $50 \%$ of both
PEDS-ED and NICU events would not have been detected and/or reported for use in QI efforts.

4.5. Smart Defibrillator and Bedside Monitor Data Collection. Over the study period, there was an increase in the proportion of CA events that had defibrillator records, defibrillator records with quality of CPR data, and bedside monitor data collected. More records were collected than had usable quality of CPR data. Defibrillator pads capable of measuring quality in patients smaller than $25 \mathrm{~kg}$ were not available until 2014, thus skewing these results. When evaluating events that had both usable CPR data from the defibrillator and bedside monitor data, this proportion increased from year to year but was still relatively low (2013: 1\%; 2014: 18\%; 2015: 27\%). As pediatric CA events are detected, the REACH system triggers patient and performance data collection mechanisms used to drive weekly debriefing of events. Over the period, both the defibrillator and bedside monitor data collection increased and were used during debriefings. For 2014 and 2015, however, 16-20\% of defibrillator records collected did not contain the quality of CPR data (Figure 5). Methods to collect bedside monitor were not available until the end of 2013. Both methods and processes were formalized in early 2014 resulting in approximately $50 \%$ of events having monitor data collected. 
TABLE 1: Counts and percentages of aggregate event, notifications, and cardiac arrest by care area.

\begin{tabular}{|c|c|c|c|}
\hline Care area & Notifications $(n=2,986)$ & Events $(n=2,145)$ & Cardiac arrest $(n=317)$ \\
\hline Clinic & $\begin{array}{c}42 \\
(1 \%)\end{array}$ & $\begin{array}{c}35 \\
(2 \%)\end{array}$ & $\begin{array}{c}0 \\
(0 \%)\end{array}$ \\
\hline Floor & $\begin{array}{l}1,030 \\
(34 \%)\end{array}$ & $\begin{array}{c}512 \\
(24 \%)\end{array}$ & $\begin{array}{c}9 \\
(3 \%)\end{array}$ \\
\hline Imaging-diagnostic & $\begin{array}{c}43 \\
(1 \%)\end{array}$ & $\begin{array}{c}19 \\
(1 \%)\end{array}$ & $\begin{array}{c}7 \\
(2 \%)\end{array}$ \\
\hline NICU & $\begin{array}{c}225 \\
(8 \%)\end{array}$ & $\begin{array}{c}170 \\
(8 \%)\end{array}$ & $\begin{array}{c}52 \\
(16 \%)\end{array}$ \\
\hline Non-children's hospital & $\begin{array}{c}51 \\
(2 \%)\end{array}$ & $\begin{array}{c}33 \\
(2 \%)\end{array}$ & $\begin{array}{c}5 \\
(2 \%)\end{array}$ \\
\hline Other children's hospital & $\begin{array}{c}50 \\
(2 \%)\end{array}$ & $\begin{array}{c}40 \\
(2 \%)\end{array}$ & $\begin{array}{c}8 \\
(3 \%)\end{array}$ \\
\hline PACU & $\begin{array}{c}113 \\
(4 \%)\end{array}$ & $\begin{array}{c}85 \\
(4 \%)\end{array}$ & $\begin{array}{c}3 \\
(1 \%)\end{array}$ \\
\hline PEDS-ED & $\begin{array}{c}365 \\
(12 \%)\end{array}$ & $\begin{array}{c}315 \\
(15 \%)\end{array}$ & $\begin{array}{c}64 \\
(20 \%)\end{array}$ \\
\hline PEDS-OR & $\begin{array}{c}84 \\
(3 \%)\end{array}$ & $\begin{array}{c}82 \\
(4 \%)\end{array}$ & $\begin{array}{c}26 \\
(8 \%)\end{array}$ \\
\hline PICU & $\begin{array}{c}983 \\
(33 \%)\end{array}$ & $\begin{array}{c}854 \\
(40 \%)\end{array}$ & $\begin{array}{c}143 \\
(45 \%)\end{array}$ \\
\hline
\end{tabular}

$\%$ values are percent of column totals.

TABLE 2: Proportion of notifications and events that are CA-related by care area.

\begin{tabular}{|c|c|c|}
\hline Care area & $\begin{array}{c}\text { Notifications that are cardiac arrest- } \\
\text { related/notifications (\%) }\end{array}$ & Events that are cardiac arrest-related/events (\%) \\
\hline Clinic & $\begin{array}{l}0 / 42 \\
(0 \%)\end{array}$ & $\begin{array}{l}0 / 35 \\
(0 \%)\end{array}$ \\
\hline Floor & $\begin{array}{c}32 / 1030 \\
(3 \%)\end{array}$ & $\begin{array}{l}9 / 512 \\
(2 \%)\end{array}$ \\
\hline Imaging-diagnostic & $\begin{array}{l}17 / 43 \\
(40 \%)\end{array}$ & $\begin{array}{c}7 / 19 \\
(37 \%)\end{array}$ \\
\hline NICU & $\begin{array}{l}96 / 225 \\
(43 \%)\end{array}$ & $\begin{array}{c}52 / 170 \\
(31 \%)\end{array}$ \\
\hline Non-children's hospital & $\begin{array}{l}11 / 51 \\
(22 \%)\end{array}$ & $\begin{array}{c}5 / 33 \\
(15 \%)\end{array}$ \\
\hline Other children's hospitals & $\begin{array}{c}8 / 50 \\
(16 \%)\end{array}$ & $\begin{array}{c}8 / 40 \\
(20 \%)\end{array}$ \\
\hline $\mathrm{PACU}$ & $\begin{array}{c}6 / 113 \\
(5 \%)\end{array}$ & $\begin{array}{l}3 / 85 \\
(4 \%)\end{array}$ \\
\hline PEDS-ED & $\begin{array}{c}80 / 365 \\
(22 \%)\end{array}$ & $\begin{array}{l}64 / 315 \\
(20 \%)\end{array}$ \\
\hline PEDS-OR & $\begin{array}{l}27 / 84 \\
(32 \%)\end{array}$ & $\begin{array}{l}26 / 82 \\
(32 \%)\end{array}$ \\
\hline $\mathrm{PICU}$ & $\begin{array}{c}216 / 983 \\
(22 \%)\end{array}$ & $\begin{array}{c}143 / 854 \\
(17 \%)\end{array}$ \\
\hline Total & $\begin{array}{c}493 / 2986 \\
(17 \%)\end{array}$ & $\begin{array}{c}317 / 2145 \\
(15 \%)\end{array}$ \\
\hline
\end{tabular}

\section{Discussion}

In 2015, the National Academies described a framework for "improving patient outcomes from cardiac arrest." This framework rests on a foundation of comprehensive surveillance and reporting underpinned by reliable and accurate data [17]. Several national-level registries exist in the United States, where data for both in-hospital and out-ofhospital CA can be reported, aggregated, and analyzed [19-21]. These have increased capacity in the resuscitation
QI and science fields by way of access to resources (the registries themselves) and the generation of reports for users and researchers. The design of these registries is informed by best practices and based on published standards such as the Utstein templates for resuscitation registries [22-25]. Although these design features help to ensure that the data submitted are standardized and can be rigorously analyzed, they do little to ensure that all eligible events from contributing institutions are detected and their data collected and submitted. This is especially reflected in the variability in 
TABLE 3: Notifications per event by care area and year, stratified by cardiac arrest status.

\begin{tabular}{lcccc}
\hline & 2013 & 2014 & 2015 & Total \\
\hline Cardiac arrest event & & & & \\
Clinic & - & - & - & - \\
Floor & 2.5 & - & $\mathbf{4 . 4}$ & 3.6 \\
Imaging-diagnostic & 2.3 & - & 2.5 & 2.4 \\
NICU & $\mathbf{1 . 5}$ & 1.8 & $\mathbf{2 . 1}$ & 1.8 \\
Non-children's hospital & 3.0 & 1.0 & 1.0 & 2.2 \\
Other children's hospitals & 1.0 & 1.0 & 1.0 & 1.0 \\
PACU & - & 2.0 & - & 2.0 \\
PEDS-ED & 1.2 & 1.3 & 1.3 & 1.3 \\
PEDS-OR & 1.0 & 1.0 & 1.1 & 1.0 \\
PICU & $\mathbf{1 . 3}$ & $\mathbf{1 . 4}$ & $\mathbf{1 . 7}$ & 1.5 \\
Total & 1.4 & 1.4 & $\mathbf{1 . 8}$ & $\mathbf{1 . 6}$ \\
Noncardiac arrest event & & & & \\
Clinic & 1.4 & 1.2 & 1.0 & 1.2 \\
Floor & 1.8 & 2.0 & $\mathbf{2 . 1}$ & 2.0 \\
Imaging-diagnostic & 1.0 & 2.1 & 2.5 & 2.2 \\
NICU & $\mathbf{1 . 1}$ & 1.2 & $\mathbf{1 . 0}$ & 1.1 \\
Non-children's hospital & 1.6 & 1.4 & 1.3 & 1.4 \\
Other children's hospitals & 1.2 & 1.8 & 1.1 & 1.3 \\
PACU & 1.3 & 1.5 & 1.1 & 1.3 \\
PEDS-ED & 1.1 & 1.1 & 1.2 & 1.1 \\
PEDS-OR & 1.1 & 1.0 & 1.0 & 1.0 \\
PICU & $\mathbf{1 . 0}$ & $\mathbf{1 . 1}$ & $\mathbf{1 . 1}$ & 1.1 \\
Total & 1.3 & 1.4 & $\mathbf{1 . 4}$ & $\mathbf{1 . 4}$ \\
\hline
\end{tabular}

Bold indicates statistically significant difference between the value for the care area and year and its complement in the comparison of cardiac arrest status group.

TABLE 4: Proportion of CA events detected only via implementation of the REACH surveillance system.

\begin{tabular}{lcccc}
\hline Care area & 2013 & 2014 & 2015 & Total \\
\hline Clinic & - & - & - & - \\
Floor & $0 / 4$ & & $0 / 5$ & $0 / 9$ \\
& $(0 \%)$ & - & $(0 \%)$ & $(0 \%)$ \\
Imaging-diagnostic & $0 / 3$ & - & $0 / 4$ & $0 / 7$ \\
& $(0 \%)$ & & $(0 \%)$ & $(0 \%)$ \\
NICU & $10 / 13$ & $8 / 15$ & $8 / 24$ & $26 / 52$ \\
& $(77 \%)$ & $(53 \%)$ & $(33 \%)$ & $(50 \%)$ \\
Non-children's hospital & $1 / 3$ & $1 / 1$ & $1 / 1$ & $3 / 5$ \\
& $(33 \%)$ & $(100 \%)$ & $(100 \%)$ & $(60 \%)$ \\
Other children's hospitals & $2 / 3$ & $2 / 2$ & $3 / 3$ & $\mathbf{7 / 8}$ \\
& $(67 \%)$ & $(100 \%)$ & $(100 \%)$ & $(\mathbf{8 8} \%)$ \\
PACU & - & $1 / 3$ & - & $1 / 3$ \\
& $(33 \%)$ & - & $(33 \%)$ \\
PEDS-ED & $16 / 23$ & $9 / 18$ & $6 / 23$ & $31 / 64$ \\
& $(70 \%)$ & $(50 \%)$ & $(26 \%)$ & $(48 \%)$ \\
PEDS-OR & $\mathbf{5 / 5}$ & $\mathbf{1 2 / 1 2}$ & $\mathbf{9 / 9}$ & $\mathbf{2 6 / 2 6}$ \\
& $(\mathbf{1 0 0 \% )}$ & $(\mathbf{1 0 0 \% )}$ & $(\mathbf{1 0 0 \% )}$ & $(\mathbf{1 0 0 \% )}$ \\
PICU & $\mathbf{3 4 / 3 8}$ & $\mathbf{4 0 / 5 1}$ & $\mathbf{2 8 / 5 4}$ & $\mathbf{1 0 2 / 1 4 3}$ \\
& $(\mathbf{8 9 \% )}$ & $\mathbf{( 7 8 \% )}$ & $\mathbf{( 5 2 \% )}$ & $(\mathbf{7 1 \% )}$ \\
Total & $68 / 92$ & $73 / 102$ & $55 / 123$ & $196 / 317$ \\
& $(74 \%)$ & $(72 \%)$ & $(45 \%)$ & $(62 \%)$ \\
\hline
\end{tabular}

reported incidence of IHCA and even more so with the wide range of pediatric estimates of CA $[4,5,12]$. Active and comprehensive surveillance is necessary at the individual hospital level to ensure not only both reliability and accuracy of the data being submitted but also their completeness.
Without complete event detection, the true incidence of pediatric CA will be underestimated. Furthermore, survival rate estimates may be inaccurate and systematic selection biases may exist in the larger national registries. To address these weaknesses, we report the development of a semiautomated, electronic, and multidisciplinary reporting system that vastly increased our capture of the CA events.

Our integrated data collection system shows that such underestimation can be improved with a systematic approach. This institution participates in the American Heart Association's "Get With the Guidelines-Resuscitation" (GWTG-R) national registry. While reporting to this QI initiative in 2011, there were eleven detected in-hospital pediatric CA. In 2012, this number increased to fourteen of which eight occurred in the PICU, four on the general wards (floor), one in a diagnostic area (MRI), and one in the PEDS-ED. Furthermore, according to these records there were "0 pediatric events" in February, August, September, and November. When these statistics were reviewed by the members of the CPR advisory committee and compared to other institutions, these numbers appeared to underrepresent the expected frequency of events.

Using the PICU as an example, in 2011 and 2012 there were approximately 2000 admissions annually. Current reported rates of CA in PICUs are estimated to range from $1.8 \%$ to $5.5 \%$ of admissions $[9,10,12]$ and therefore using these rates, the expected number of CA that should be observed in the PICU is between 36 and 110. In 2012, the observed number of events was 8 versus the expected of at least 36. This observation further aided in validating the concern that pediatric CA events were being missed using the methods of detection in place at the time.

After the implementation of the REACH surveillance system, the documented number of CA was closer to the expected incidence. First, the count of events identified only by previous methods was similar with historical counts (Table 4), suggesting that the system was not missing any that were previously being detected. Second, the difference between the CA events that would have been detected and what was detected show a true increase in events captured (e.g., 2013: 4 versus 38 events). Lastly, the calculated incidence based on the detected frequency of these events falls within the expected estimates as reported in the literature $[4,5,9,10,26]$. The measured incidence for PICU events (CA/admissions) was approximately $2 \%$ annually (2013: 38/2100 (1.8/100 admissions); 2014: 51/2262 (2.2/100 admissions); 2015: 54/2203 (2.5/100 admissions)), suggesting that the system was approaching $100 \%$ capture in the PICU.

The increase in detected events for the entire pediatric population by year and in previously underreported care areas indicates that the system objective to identify every pediatric CA is on its way to being met. By leveraging additional electronic sources to identify candidate events and along with a multidisciplinary team to verify CA status created a more effective system. Moreover, as comprehensive local surveillance processes or systems such as the REACH system are deployed, more accurate and reliable pediatric CA event datasets, with less selection bias, can be submitted to national registries. 


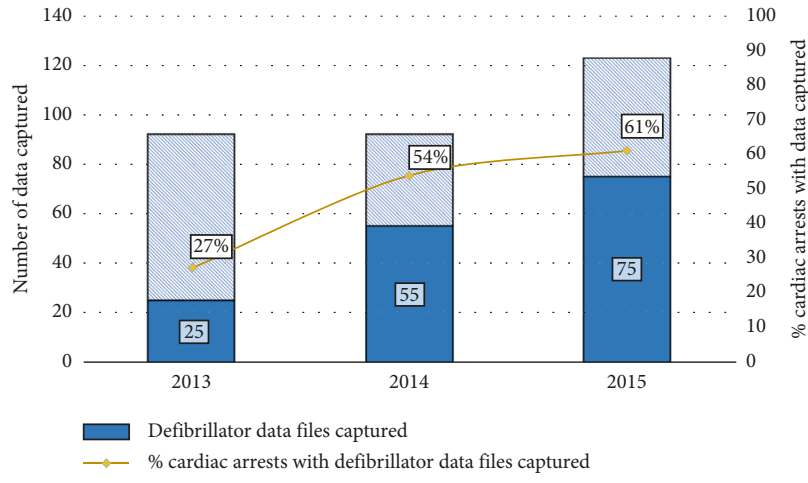

(a)

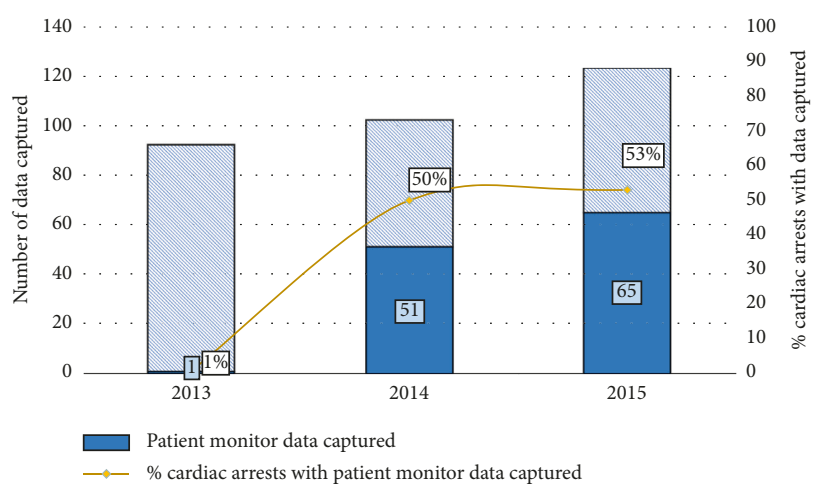

(c)

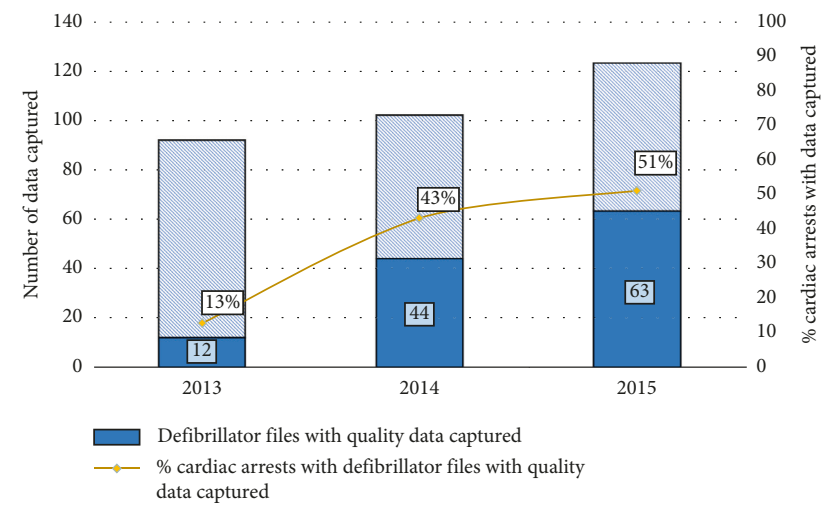

(b)

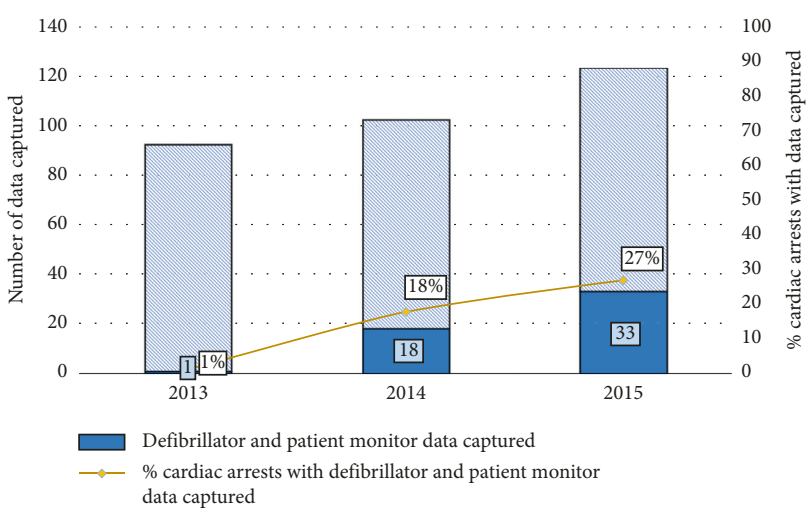

(d)

FIgURE 5: Proportion of CA events with smart defibrillator and bedside monitor data collected by year (2013-2015).

Detection of every event not only promotes the completeness, accuracy, and reliability of registry data, it also provides for more opportunities to critically and objectively debrief these events. Technological advances in recent years have allowed for (1) patient and CPR performance data capture during cardiopulmonary arrest, (2) real-time feedback, and (3) postevent evaluation of the health-care provider performance. A systematic review of these technologies suggests that their use during training helps to improve skill retention [27]. Despite the ability for these devices to provide feedback during CPR, it is unclear whether this alone is sufficient to affect the sustained healthcare provider performance [28-30]. Data collected during CPR have been shown to help improve subsequent CPR quality performance when used during cold debriefings (where individuals or teams are provided with feedback sometime after the event) [28, 31,32]. Couper and Perkins assert that objective performance data are a key requirement for cold debriefing [33] and this is particularly important for determining guideline compliance, identifying poor performance, understanding high performance, and a shared mental model of what is exquisite CPR. Furthermore, these debriefings can provide opportunities to confirm the accuracy of data that will be entered in the GWTG-R registry, as medical records are often incomplete or inaccurate [34]. In 2014 and 2015, although not every event's bedside monitor data was captured, (2014: 51/102 (50\%); 2015:
$65 / 123(53 \%))$ the ability to use the multidisciplinary team to confirm or validate various objective measures (e.g., initial rhythm, time of pulselessness, time to initiation of CPR, time from shockable rhythm to defibrillation, and use of a device to confirm endotracheal tube placement) allowed for appropriate identification of each CA event and accurate registry reporting. Siems et al. recently confirmed the highdegree of inaccuracy in documentation of the time to initiation of CPR metric [35]. Bedside data are either lost due to the patient being discharged from the monitor system, time passing and data being overwritten, or system limitations (e.g., the OR monitor system does not interface with the data collection tool used in other care areas). Highlighting the importance of this data during debriefing has motivated leadership within each care area to implement processes to ensure that the data are not discharged or deleted prior to their collection. With the addition of a project coordinator and two volunteer clinical staff to provide capacity to collect the monitor data outside daytime hours and on the weekends, the proportion of all events with bedside data captured in 2016 has increased to $72 \%$.

Although this system has increased the detection of events across the pediatric care areas, particularly in acute and critical care settings, it is still challenging to know if every event is being detected. More work is needed to collect appropriate denominator data for all care areas to effectively analyze CA incidence for comparison against similar 
institutions. Data mining of the EHR also holds the potential to determine the missed event rate, or to generate live-time alerting of CA events to be used in conjunction with or supplemental to a surveillance system such as this.

\section{Conclusion}

The implementation of a surveillance system to identify pediatric CA events, using organizationally available notification sources has resulted in an increase in CA event detection. Prior to the development of the REACH system, utmost 14 events per year were detected. After deployment, this has increased almost 9-fold to 123 pediatric CAs per year which is closer to the expected incidence. Had the system not been implemented and using predeployment methods of detection, $100 \%$ of PEDS-OR events would have been missed along with $50-70 \%$ of events from the PICU, NICU, and ED. Improved data capture through the REACH surveillance system not only provided objective assessment of guideline compliance and level of CPR quality and potential subsequent performance improvements, but they also allowed for confirmation of key data elements submitted to GWTG$\mathrm{R}$ thus improving accuracy and reliability of the overall registry. An effective surveillance system, objective performance data, and active quality improvement initiatives can drive efforts to further improvements in the quality of CPR provided to children everywhere.

\section{Conflicts of Interest}

The authors declare that they have no conflicts of interest.

\section{Appendix}

\section{Development and Configuration of System Components}

The following describes what configuration or development was needed to integrate each notification source into the REACH system.

\section{A.1. Development: Notification Sources}

(i) PING (Johns Hopkins, Baltimore). This organizationally developed web-based messaging service is used to communicate throughout the Children's Center and was harnessed for two particular classes of events that had the potential to represent a cardiac arrest. Integration of this system was achieved by the list owners (clinicians) adding the REACH email address to the recipient list for the following situations involving critically ill children:

(ii) Pediatric Expedited Transfer (PET). When a critically ill child needs an expedited transfer from the Emergency Department to the PICU, a PET team is paged.

(iii) Code Blue Button. Connexall (Connexall USA Inc., Boulder, CO). The intended use of these buttons is to summon additional help for care providers during urgent or emergency situations. When used in conjunction with a clinical telephony solution (Ascom North America, Morrisville, NC), this system allows for text-based messaging to groups of care team members through integrated call systems, clinical phones, or email. Integration was achieved by (the clinical engineer department) adding a rule that triggers an email to the REACH account when buttons are pressed.

(iv) Emergency Response Paging. SDC Intellidesk (SDC Solutions, Manchester, NH) When the Pediatric Rapid Response Team (RRT) is called for and dispatched (at this institution for both cardiac arrest and noncardiac arrest emergencies), a message is sent via page to members of the team. The paging system can also send a copy via email. Integration was achieved by (the pager administration office) adding the REACH email address to the RRT recipient list.

(v) Electronic Health Record (EHR). Sunrise Clinical Manager (Allscripts, Chicago, IL). This EHR has the capability to trigger an email when cardiac arrest or rapid response flowsheets are opened and used to document the event. Integration was achieved by (a Clinical Information System technical lead) adding a rule defining the $\mathrm{REACH}$ email address as the recipient.

(vi) Respiratory Therapy Services Billing. As part of the ongoing finance and accounting processes by the hospital's Respiratory Therapy (RT) organizational unit, a report of all billable services provided by respiratory therapists is automatically generated daily. This report contains codes for Respiratory Therapy services provided to patients. Three specific codes associated with CPR are often used: Cardiac Arrest, Intubation Assist, and Manual Ventilation. This report is sent from the billing system as a comma-separated values (CSVs) file to the RT manager. The manager created an email inbox rule to forward this to the REACH email address.

(vii) Virtual Code Blue (VCB) Button. Prior to the development of the VCB button, when no notification source was activated, but a member of the MDQI group knew of a pediatric cardiac arrest, a manual entry would be made via the User Interface by a system administrator. The VCB notification allows for the same functionality through a secure web-based form and by any member of the organization. This form creates a notification and inserts it directly into the database, while simultaneously initiating the data collection process. This was developed as part of the System Logic and User Interface application.

A.2. Development: Organizational Enterprise Email. Microsoft Exchange Server (Microsoft, Redmond, WA) is this institution's enterprise email system. No custom 
development or server-level modifications were necessary. A dedicated email account was created, which required a formal request to system administrators. A feature known as "inbox rules" allows for an end-user to define a number of automated tasks to be performed on messages as they arrive. This feature was leveraged to identify organizational IT system-generated notifications and forward these to the MDQI listserv. Determining valid emails consisted of one or more conditions based on the sender email address and known keywords being satisfied.

A.3. Development: Database. The database solution used is Microsoft SQL Server Express Edition (64-bit) Version 10. It consists of 20 tables that provide data storage and relationship definitions. These provide storage and structure to standardize all IT systems notification data from free-text message formats to that which allows for the creation of relationships between notifications, events (including type and location), and collected smart defibrillator and bedside monitor data.

A.4. Development: System Logic and User Interface. The System Logic and User Interface were developed using ASP. NET 4.5 Web Forms (C\#) and runs on Internet Information Services (IIS) version 7.5. This application requires an SSL connection, using a SHA-256, RSA (2048 bit) certificate. This application is accessible only while on the institutional network or connected remotely through a secure VPN connection, using a fully qualified domain name managed by the institutional DNS. Authentication is also managed using the institutional single sign-on (SSO) service, whereas authorization is managed by the system database. Web forms were developed to provide for management of notification, event, and smart defibrillator and bedside monitor data, as well as data export and analysis.

A.5. Development: Listserv. The listserv is provided as an institutional communication tool powered by Sympa (RENATER. Paris, France). This tool is configured such that only the REACH email service and list members can send to it, and that any reply is a reply-all.

A.6. Development: Notification Polling Service. The Notification Polling Service is a Windows Service Application, developed in C\# and running on an institutional server. This application uses the Exchange Web Services Managed API v2.0 (Microsoft, Redmond, WA) to interact with the REACH mailbox and a data access library providing database access. This service checks for new messages every 10 seconds and manages previous interactions with the system through the preexisting "read-unread" built-in feature of Exchange as well as a check of the message's GUID presence in the database. This allows for MS Exchange, the database, or the polling service to be offline for any number of reasons, but resume normal operation (particularly processing of notifications and creation of events) as soon as all are available.

\section{References}

[1] G. Nichol, E. Thomas, C. W. Callaway et al., "Regional variation in out-of-hospital cardiac arrest incidence and outcome," JAMA, vol. 300, no. 12, pp. 1423-1431, 2008.

[2] A. S. Go, D. Mozaffarian, V. L. Roger et al., "Heart disease and stroke statistics-2013 update: a report from the American Heart Association," Circulation, vol. 127, no. 1, pp. e6-e245, 2013.

[3] R. M. Merchant, L. Yang, L. B. Becker et al., "Incidence of treated cardiac arrest in hospitalized patients in the United States," Critical Care Medicine, vol. 39, no. 11, pp. 2401-2406, 2011.

[4] P. S. Chan, R. Jain, B. K. Nallmothu, R. A. Berg, and C. Sasson, "Rapid response teams: a systematic review and meta-analysis," Archives of Internal Medicine, vol. 170, no. 1, pp. 18-26, 2010.

[5] L. J. Morrison, R. W. Neumar, J. L. Zimmerman et al., "Strategies for improving survival after in-hospital cardiac arrest in the United States: 2013 consensus recommendations: a consensus statement from the American Heart Association," Circulation, vol. 127, no. 14, pp. 1538-1563, 2013.

[6] A. G. Reis, V. Nadkarni, M. B. Perondi, S. Grisi, and R. A. Berg, "A prospective investigation into the epidemiology of in-hospital pediatric cardiopulmonary resuscitation using the international Utstein reporting style," Pediatrics, vol. 109, no. 2, pp. 200-209, 2002.

[7] A. D. Slonim, K. M. Patel, U. E. Ruttimann, and M. M. Pollack, "Cardiopulmonary resuscitation in pediatric intensive care units," Critical Care Medicine, vol. 25, no. 12, pp. 1951-1955, 1997.

[8] P. Suominen, K. T. Olkkola, V. Voipio, R. Korpela, R. Palo, and J. Räsänen, "Utstein style reporting of in-hospital paediatric cardiopulmonary resuscitation," Resuscitation, vol. 45, no. 1, pp. 17-25, 2000.

[9] R. Ronco, W. King, D. K. Donley et al., "Outcome and cost at a children's hospital following resuscitation for out-ofhospital cardiopulmonary arrest," Archives of Pediatrics \& Adolescent Medicine, vol. 149, no. 2, pp. 210-214, 1995.

[10] M. M. Bembea, V. M. Nadkarni, M. Diener-West et al., "Temperature patterns in the early postresuscitation period after pediatric inhospital cardiac arrest," Pediatric Critical Care Medicine, vol. 11, no. 6, pp. 723-730, 2010.

[11] K. L. Meert, A. Donaldson, V. Nadkarni et al., "Multicenter cohort study of in-hospital pediatric cardiac arrest," Pediatric Critical Care Medicine, vol. 10, no. 5, pp. 544-553, 2009.

[12] N. de Mos, R. R. van Litsenburg, B. McCrindle, D. J. Bohn, and C. S. Parshuram, "Pediatric in-intensive-care-unit cardiac arrest: incidence, survival, and predictive factors," Critical Care Medicine, vol. 34, no. 4, pp. 1209-1215, 2006.

[13] A. G. Randolph, C. A. Gonzales, L. Cortellini, and T. Yeh, "Growth of pediatric intensive care units in the United States from 1995 to 2001," Journal of Pediatrics, vol. 144, no. 6, pp. 792-798, 2004.

[14] N. Garber, R. S. Watson, W. T. Linde-Zwirble et al., "The size and scope of intensive care for children in the US," Critical Care Medicine, vol. 31, p. A78, 2003.

[15] S. Girotra, J. A. Spertus, Y. Li et al., "Survival trends in pediatric in-hospital cardiac arrests: an analysis from get with the guidelines-resuscitation," Circulation: Cardiovascular Quality and Outcomes, vol. 6, no. 1, pp. 42-49, 2013.

[16] R. A. Berg, R. M. Sutton, R. Holubkov et al., "Ratio of PICU versus ward cardiopulmonary resuscitation events is increasing," Critical Care Medicine, vol. 41, no. 10, pp. 22922297, 2013. 
[17] R. Graham, M. A. McCoy, A. M. Schultz et al., Strategies to Improve Cardiac Arrest Survival: A Time To Act, The National Academies Press, Washington, DC, USA, 2015, http://www. nap.edu/catalog/21723/strategies-to-improve-cardiac-arrestsurvival-a-time-to-act.

[18] F. Davis, "Perceived usefulness, perceived ease of use, and user acceptance of information technology," MIS Quarterly, vol. 13, no. 3, pp. 319-340, 1989.

[19] D. P. Davis, L. A. Garberson, D. L. Andrusiek et al., "A descriptive analysis of emergency medical service systems participating in the resuscitation outcomes consortium (ROC) network," Prehospital Emergency Care, vol. 11, no. 4, pp. 369-382, 2007.

[20] B. McNally, A. Stokes, A. Crouch, and A. L. Kellermann, "CARES: cardiac arrest registry to enhance survival," Annals of Emergency Medicine, vol. 54, no. 5, pp. 674-683, 2009.

[21] R. M. Merchant, L. Yang, L. B. Becker et al., "Variability in case-mix adjusted in-hospital cardiac arrest rates," Medical Care, vol. 50, no. 2, pp. 124-130, 2012.

[22] I. Jacobs, V. Nadkarni, J. Bahr et al., "Cardiac arrest and cardiopulmonary resuscitation outcome reports: update and simplification of the Utstein templates for resuscitation registries," Circulation, vol. 63, no. 3, pp. 233-249, 2004.

[23] R. O. Cummins, D. A. Chamberlain, N. S. Abramson et al., "Recommended guidelines for uniform reporting of data from out-of-hospital cardiac arrest: the Utstein Style, a statement for health professionals from a task force of the American Heart Association, the European Resuscitation Council, the Heart and Stroke Foundation of Canada, and the Australian Resuscitation Council," Circulation, vol. 84, no. 2, pp. 960-967, 1991.

[24] A. Zaritsky, V. Nadkarni, M. F. Hazinski et al., "Recommended guidelines for uniform reporting of pediatric advanced life support: the pediatric Utstein Style," Circulation, vol. 92, no. 7, pp. 2006-2020, 1995.

[25] R. O. Cummins, D. Chamberlain, M. F. Hazinski et al., "Recommended guidelines for reviewing, reporting, and conducting research on in-hospital resuscitation: the inhospital "Utstein style"," Resuscitation, vol. 34, no. 2, pp. 151-183, 1997.

[26] V. M. Nadkarni, G. L. Larkin, M. A. Peberdy et al., "First documented rhythm and clinical outcome from in-hospital cardiac arrest among children and adults," JAMA, vol. 295, no. 1, pp. 50-57, 2006.

[27] J. Yeung, R. Meeks, D. Edelson, F. Gao, J. Soar, and G. D. Perkins, "The use of CPR feedback/prompt devices during training and CPR performance: a systematic review," Resuscitation, vol. 80, no. 7, pp. 743-751, 2009.

[28] D. P. Edelson, B. Litzinger, B. S. Abella et al., "Improving inhospital cardiac arrestprocess and outcomes with performance debriefing," Archives of Internal Medicine, vol. 168, no. 10, pp. 1063-1069, 2008.

[29] B. S. Abella, D. P. Edelson, S. Kim et al., "CPR quality improvement during in-hospital cardiac arrest using a real-time audiovisual feedback system," Resuscitation, vol. 73, no. 1, pp. 54-61, 2007.

[30] A. Bohn, T. P. Weber, S. Wecker et al., "The addition of voice prompts to audiovisual feedback and debriefing does not modify CPR quality or outcomes in out of hospital cardiac arrest: a prospective, randomized trial," Resuscitation, vol. 82, no. 3, pp. 257-262, 2011.

[31] C. Jiang, Y. Zhao, Z. Chen, S. Chen, and X. Yang, "Improving cardiopulmonary resuscitation in the emergency department by real-time video recording and regular feedback learning," Resuscitation, vol. 81, no. 12, pp. 1664-1669, 2010.
[32] H. A. Wolfe, R. M. Sutton, J. Leffelman et al., "Quantitative post-cardiac arrest audiovisual debriefing improves resuscitation quality," Circulation, vol. 126, no. 21, p. A137, 2012.

[33] K. Couper and G. D. Perkins, "Debriefing after resuscitation," Current Opinion in Critical Care, vol. 19, no. 3, pp. 188-194, 2013.

[34] K. Couper and B. S. Abella, "Auditing resuscitation performance: innovating to improve practice," Resuscitation, vol. 83, no. 10, pp. 1179-1180, 2012.

[35] A. Siems, E. Tomaino, A. Watson, M. C. Spaeder, and L. Su, "Improving quality in measuring time to initiation of CPR during in-hospital resuscitation," Resuscitation, vol. 118, pp. 15-20, 2017. 


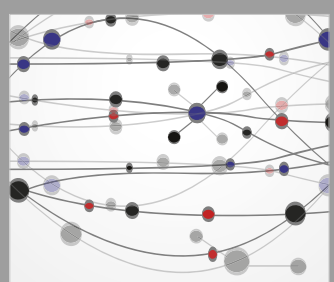

The Scientific World Journal
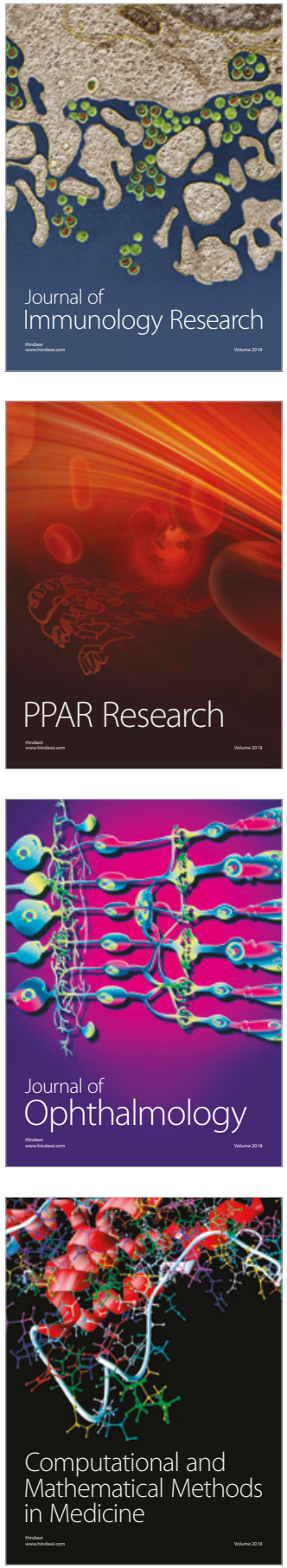

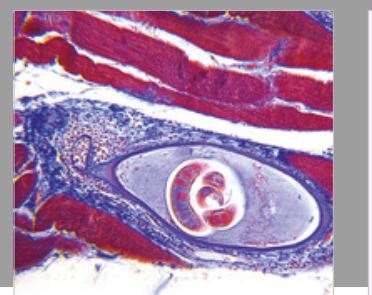

Gastroenterology Research and Practice

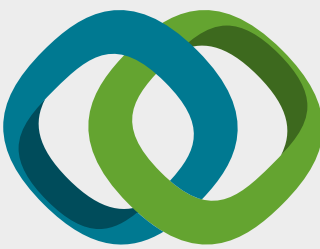

\section{Hindawi}

Submit your manuscripts at

www.hindawi.com
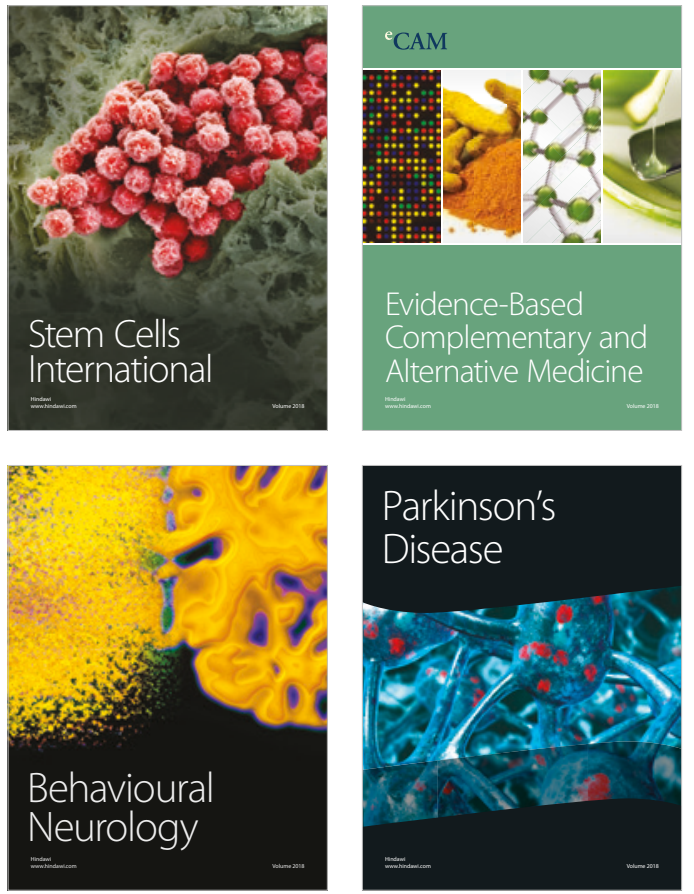

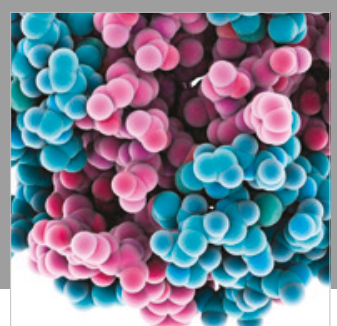

ournal of

Diabetes Research

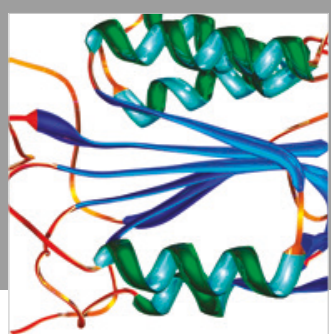

Disease Markers
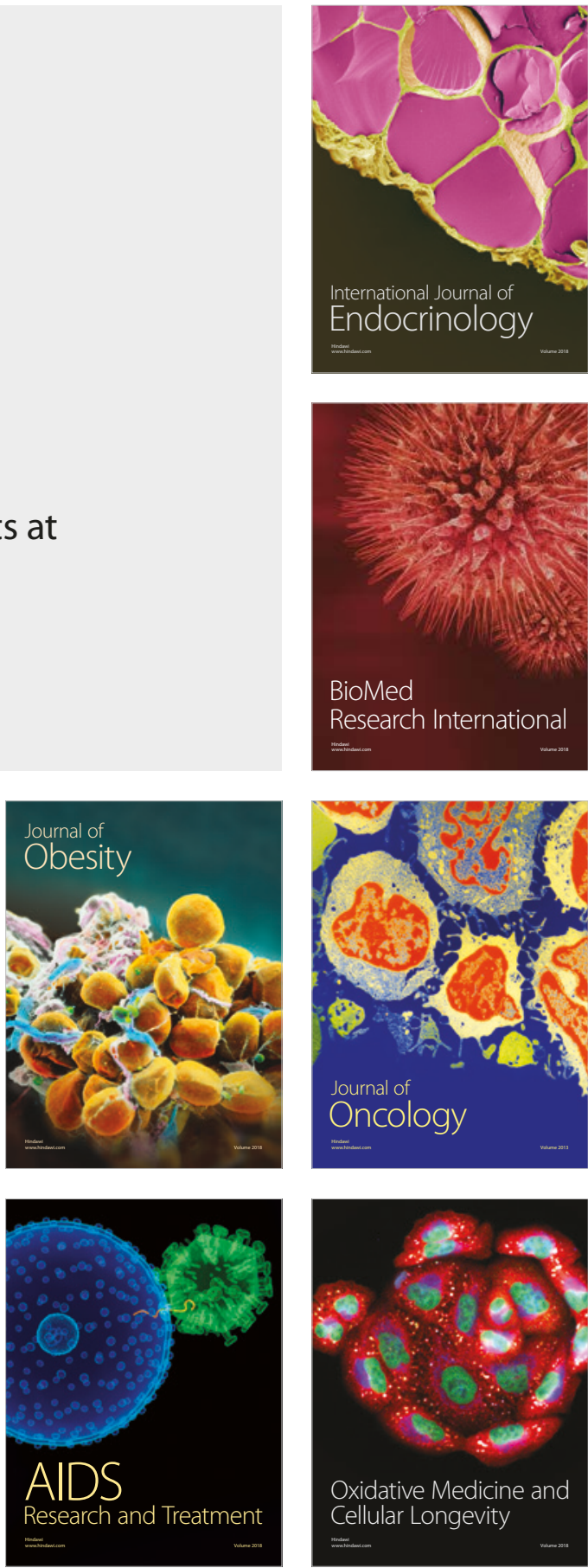